\title{
Lesion Revascularization Ischemia Indicator
}

National Cancer Institute

\section{Source}

National Cancer Institute. Lesion Revascularization Ischemia Indicator. NCI Thesaurus.

Code C119555.

An indication as to whether clinical or functional ischemia is present before lesion

revascularization occurred. 